\title{
The worldwide challenge on myopia
}

In these conditions half of the last century, two notable epidemiologists (Omran and Popkin) $)^{1,2}$ systematized statistic information, formulated original interpretations and consolidated foundations of a new descriptive perspect and a quick, surprising and changing process in analyzing morbidity/mortality indicators, characterizing the so-called epidemiological transition. Since then, researchers, governamental healthcare agents and research institutions from various countries began to unite temporal and geographic trends indicatings triking alterations in traditional patterns of health/disease at a population level in different fields of observation.1,2

With this alternative view on the epidemiological transition, several questions have entered in space of new and systematic atypias references and the combination of diseases, innovativing amazing applications, such as epicdemic cesarean, the premature weaning and its adverse implications for the binomial mother/child, the derived introgens is from diagnostic procedures, clinical treatments, surgical and even "hygienist" when a possible exaggeration of promoting care and health by protecting children in which begins to store present biological conditions, vulnerability hither where would never be suspected. 3

In this new order of speculation, myopia begins to increase a worrisome behavior in registering services and the intervention on the population health scale and its new demands in the intra and extra-sectoral area of the individual and collective health. This is an issue that has become evident and disturbing visibility for the last five years by justifying the six health regions convention that formed the technical-administrative jurisdiction in the World Health Organization (WHO).

In fact, the speed and magnitude of myopia expansion in a population level was surprisingly readiness in responding international organizations, ahead of the WHO itself and its committees of "experts". Thus, welcoming the $6^{\text {th }}$ Health Region in Australia justifies all the continents and countries in becoming a universal agenda by discussing potential demands in which became disturbed themes and being a priority in various nations in the world. Evidence is consistent in itself, currently $33 \%$ of the population in the world already constitutes of myopia prevalence as a well characterized public health problem. 4

Moreover, the construction of temperal series, gathering retrospective data and their prospective projections, makes it possible to characterize a challenge and an "even dramatic" situation, given by its size, and severe consequences (visual impairment and blindness) and the structure of nature in its factors and effects, supported by behavioral trends and demands of modernity itself. Thus, it is conventional to a starting point in the beginning of the $21^{\text {st }}$ century (in the year 2000) when the estimated myopia prevalence was already $22 \%$ worldwide, as it was already quite high, its occurrence grew to $28 \%$ in 2010 , increasing to $33 \%$ in 2019 , estimating at $46 \%$ in the next 10 years (in 2040) and finally 52\% in 2050. In other words, soon, a little over twenty years from now, more than half of the population will present fractive disorder that is defined as myopia. ${ }^{4}$

Assuming all the characteristics of a progressive epidemy, as an organic and functional problem that within a hundred years, counting and adding its retrospective statistics, predictively, projecting of its growth in the next three or four decades, myopia will gain an unusual dimension in history as any other human nosography. Extrapolating its traditional setting in the genetic dominance, as myopia now assumes the etiopathogeny of a severe environmental disorder in the sense of abnormality and complex relationship of man/ecosystem. 5,6 
From a singular refractive error that could accommodate an occurrence of 5-10\%, is still known as an epidemiological event in many rural communities or urban agglomerations in various countries in the world, myopia was settled for inhabitants, the physical working environment, space, time, study and work practices, the outdoor weather and the views of the aquactic horizons, such as rivers, lakes, seas and oceans, or lands, as the mountains or the "endless lands" of large geographic plains are progressively limited or abolished by small chambers to the human eye. Currently, much of the population, the visual domain and the operational manuals are performed at a distance of $25 \mathrm{~cm}$ or less, conditioned to limits at a distance that does not pass the borders of the so-called "the tip of the nose". By the way, these short distances have been studied with unhealthy factors of the function of the eye in a diverse but restricted fields of chores and pleasures to the sight. Population studies, clinical histories and countless experimental trials are confluent in this sense, defining the small world to the larger or greater of all epidemies nowadays, as myopia. The problem is already characterized. The challenge now is a difficult effective mobilizing policies and ideas for its control and, within the limits of available knowledge for its solution. It is a challenge that questions the behavior that characterizes the current modernity.

With a scope that covers the aspects of Women's Health, not only clinical but also epidemiological, The Brazilian Journal of Maternal Child Health is open to diffuse studies that address this issue giving its importance to the Public health now and in years to come.

\section{Acknowledgements}

The author would like to thank Camila Carvalho and Déborah Lemos from the Nutrition Research Group at IMIP for their invaluable collaboration in their work in consulting and summarizing bibliographic sources and the various corrections of this text that briefly portraits the challenging perspectives of a new epidemy.

\section{References}

1. Omran AR. The epidemiologic transition: A theory of the epidemiology of population change. Milbank Q. 2005.

2. Popkin BM. Nutritional Patterns and Transitions. Popul Dev Rev. 2006; 19 (1): 138.

3. Batista Filho M, Santos CC. Cesarean section surgeries: the temporal evolution, epidemic tendency, the WHO proposals and recommendations, current doubts and challenges. Rev Bras Saúde Matern Infant. 2018; 18 (4): 851-4.

4. WHO. The Impact of Myopia and High Myopia: Report of the Joint World Health Organization-Brien Holden Vision Institute Global Scientific Meeting on Myopia. University of New South Wales, Sydney, Australia, 16-18 March 2015.; 2017.

5. Rudnicka AR, Kapetanakis VV, Wathern AK, Logan NS, Gilmartin B, Whincup PH, Cook DG, Owen CG. Global variations and time trends in the prevalence of childhood myopia, a systematic review and quantitative meta-analysis: Implications for aetiology and early prevention. Br J Ophthalmol. 2016; 100 (7): 882-90.

6. Thorn F, Cruz AA V, Machado AJ, Carvalho RAC. Refractive status of indigenous people in the northwestern Amazon region of Brazil. Optom Vis Sci. 2005; 82 (4): 267-72.

Malaquias Batista Filho 1

(iD https://orcid.org/0000-0002-1490-0590

1 PhD in Public Health. CNPq Fellow 1-A Level. Professor and researcher at the Instituto de Medicina Integral Prof. Fernando Figueira. Rua dos Coelhos, 300. Boa Vista. Recife, PE, Brazil. CEP: 50.070-902. 